\title{
Model Reference Sliding Mode Control for Bioreactor Benchmark Problem
}

\author{
Mehmet Önder Efe
}

\begin{abstract}
Process engineering is an interesting field for control researchers as the problems are based on the physics and chemistry of continuously operating and nonlinear phenomena. Oftentimes the models of processes are nonlinear and are composed of few differential equations displaying a rich set of responses. This paper considers the sliding mode control of a biochemical process within the context of model reference control. The considered process was introduced as a benchmark process displaying several challenges making it an interesting test bed. The cell mass of the process is desired to follow the output of a first order reference model, and a sliding mode controller is designed to observe the model following property. The results demonstrate that the system responds quickly and converges accurately towards what is prescribed.
\end{abstract}

\section{INTRODUCTION}

The desire of obtaining a good closed loop response in chemical process engineering is a core issue entailing a thorough investigation of the process to be controlled. In many instances, the process dynamics is described by few ordinary differential equations yet the presence of limit cycles and the inextricably intertwined nature of the process variables make it difficult. One alternative is to linearize the system dynamics then to utilize the tools of classical control theory, however, the performance obtained through linear control actions is typically limited and sometimes unsatisfactory. Nonlinear control methods can provide better closed loop responses and this aspect of chemical processes make them good test beds for benchmarking. A review of nonlinear control techniques on chemical processes is presented in [1], where the feasibility and efficacy of nonlinear control laws are discussed with an emphasis on relevant control challenges displayed by chemical processes.

In [2], Ungar defines a Bioreactor Benchmark Problem that excellently fits in the context. The state of the process is described by two dimensionless variables named the cell mass denoted by $c_{1}$ and the amount of nutrients denoted by $c_{2}$, (See [2], [3] and [4], [5]). The goal is to maintain the cell mass at desired levels by altering the inflow rate at a rate equal to outflow rate keeping the reaction volume constant. The challenges associated with the control of this process are the nonlinearity enabling the emergence of a rich set of dynamical regimes, instabilities caused even by tiny variations in the process variables and the presence of a long control sampling interval in the feedback loop.

In the past, this process was used several times for feedback control purposes. [4] consider this problem for de-

Dr. Efe is with TOBB Economics and Technology University, Department of Electrical and Electronics Engineering, Sögütözü, Ankara, Turkey, Phone: +90-312-292-4064, Fax: +90-312-292 4180 onderefe@ieee. org veloping a nonlinear control law forcing the process states to those of a first order linear one. Feedforward neural networks are used to build the nonlinear function in the control law and the plant is forced to follow a reference model. Feldkamp et al, [3], study this problem in the context of demonstrating the efficacy of a neural network learning algorithm and consider the control problem about a setpoint in the stable region, another setpoint in the unstable region and a transition between these regions. The controller proposed in our study forces the system toward the stable region as will be discussed in the sequel. Brengel et al, [6], propose a multi step nonlinear controller based on predictive control theory and validate the performance of the closed loop control system on a variant of the model considered here. The authors emphasize the preferability of operating at highest possible cell mass solutions, which are desired to be reasonably away from the region of periodic oscillations. In [7], it is emphasized that the controller design for the bioreactor benchmark problem addressed here is a challenge due to the nonlinearity and the a set of complicated regimes that arise due to it. Clearly, the works mentioned above motivate us to position the merit and effectiveness of sliding mode control techniques in the control of continuously stirred tank reactors. In this paper, we consider the problem of model following in the cell mass and we analyze the limitations of the design carried out with a thorough discussion.

Sliding Mode Control (SMC), also known as Variable Structure Control, is a well established approach ensuring some degrees of robustness against uncertainties in the feedback loop. The underlying idea is to create a sliding subspace, which is an attractor due to the philosophy of the design, [8], [9]. SMC technique, which has many successful applications in motion control systems, is also applied for feedback control of chemical processes. See for example [10], where the process is modeled by a partial differential equation, [11], where the design is based on a first order model including dead time, and [12], where a second order sliding mode control is performed after feedback linearization. One fact in all these studies needs emphasis: The sliding mode controller drives the system toward the sliding manifold and maintains the evolution on that loci, which is stable by the design and the error converges the origin of the phase space. Once the trajectories are confined to the sliding manifold, the control system displays some degrees of robustness against disturbances and parameter variations in system dynamics. This response is called invariance property of sliding mode control, [8], [13]. The underlying idea in this paper is to implement a robust model reference controller 


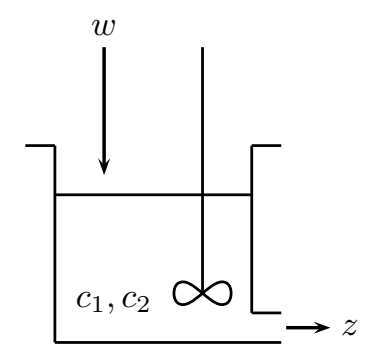

Fig. 1. Reaction tank with equal inflow and outflow rates

for the bioreactor benchmark problem. The reaching law approach in [8] is followed in postulating the control law.

This paper is organized as follows: The second section introduces the bioreactor benchmark problem and analyzes its behavior. Next we present the design of the model reference sliding mode control. The fourth section is devoted to the operating conditions, simulation results and their interpretation. The concluding remarks are given at the end of the paper.

\section{Bioreactor Benchmark Problem}

The bioreactor is a tank in which the biological cells are mixed with nutrients and water as shown in Fig. 1. The cells and nutrients are in a dynamical interaction modeled by (1) and (2), where $c_{1}$ denotes the cell mass while $c_{2}$ stands for the nutrient amount. The process is continuously fed by pure water and the variable characterizing the inflow rate is denoted by $w$. In order to maintain the reaction volume constant, the contents of the tank is removed at a rate equal to the inflow rate, $z$, which is composed of a mixture of cells, nutrients and water. The goal of the control problem is to achieve the tracking of a desired temporal evolution in cell mass. The state variables of the process and limited numerical information about the nonlinearities seen below are assumed to be available for constructing the controller.

$$
\begin{gathered}
\dot{c}_{1}=-c_{1} w+c_{1}\left(1-c_{2}\right) e^{\frac{c_{2}}{\gamma}} \\
\dot{c}_{2}=-c_{2} w+c_{1}\left(1-c_{2}\right) e^{\frac{c_{2}}{\gamma}} \frac{1+\beta}{1+\beta-c_{2}}
\end{gathered}
$$

where the state variables are constrained by $\Omega:=0 \leq$ $c_{1}, c_{2} \leq 1$. In the nominal plant model given above, the growth rate is characterized by the parameter $\beta=0.02$ and the nutrient inhibition parameter is given by $\gamma=0.48$.

In Fig. 2, several trajectories are shown for a set of initial conditions denoted by a circle. Each subplot depicts the evolution of the system at a constant inflow rate indicated on the top. Depending on the value of the inflow rate, the attractors change their locations and new attractors emerge as well. One visible one is a limit cycle which becomes apparent when $w=0.75$. When $w=0.8290$, the system changes its qualitative behavior radically. Computing the equilibrium values corresponding to this inflow rate, one obtains $c_{1}=0.1331$ and $c_{2}=0.8626$. The eigenvalues of the linearized system of equations at this point stipulate that in the increasing direction of $c_{2}$, the system undergoes Hopf bifurcation at this operating point and turns into an unstable one displaying spontaneous oscillations due to the limit cycle. In this regime, cell mass varies in between 0.1219 and 0.1466 while the nutrient amount fluctuates in between 0.8243 and 0.8996 . At the points of crossing the imaginary axis, the eigenvalues of the linearized model are approximately equal to $0 \pm j 1.7543$, from which we infer that the self sustained oscillations are quite fast.

In Fig. 3, the limit cycle and the convergence of the neighboring trajectories are illustrated for $w=1.2$. In fact, limit cycles can occur for all values of admissible inflow rates, i.e. $0 \leq w \leq 2$. According to Bendixson theorem (See [14], [15]), since the quantity

$$
\begin{aligned}
H:= & \frac{\partial}{\partial c_{1}}\left(-c_{1} w+c_{1}\left(1-c_{2}\right) e^{\frac{c_{2}}{\gamma}}\right)+ \\
& \frac{\partial}{\partial c_{2}}\left(-c_{2} w+c_{1}\left(1-c_{2}\right) e^{\frac{c_{2}}{\gamma}} \frac{1+\beta}{1+\beta-c_{2}}\right) \\
= & -2 w+h\left(c_{1}, c_{2}\right)
\end{aligned}
$$

does not vanish and does not change sign in $\Lambda \subseteq \Omega$, no limit cycles can exist entirely in $\Lambda$. For a given constant $w$, the curve of sign change for $H$ is moved to the curve described by $h\left(c_{1}, c_{2}\right)=2 w$. Therefore one should run the quantity $2 w$ from 0 to 4 and determine where the sign change occurs. In Fig. 4, the regions where the limit cycles cannot lie entirely within are depicted as white regions, termed $\Lambda$ above, and the value of $2 w$ is contoured for $2 w$ equals to $0,2,3$ and 4 . According to this result, we figure out that it is possible to have other limit cycle trajectories in the system dynamics and $\Lambda$ is a significantly wide subspace of $\Omega$. From the control engineering point of view, this practically tells us that during the controlled operation of the process, many attractors and/or repellers can be created or destroyed depending on the value of $w$ and the controller must be overcoming the dynamical influence of such difficulties while meeting the performance specifications and revealing disturbance rejection.

Consider the process at the steady state, i.e. $\dot{c}_{1}=0$ and $\dot{c}_{2}=0$. This yields the steady state control action $w_{s s}=\left(1-c_{2}\right) e^{\frac{c_{2}}{\gamma}}$ and with this control signal $\dot{c}_{2}=$ $\left(1-c_{2}\right) e^{\frac{c_{2}}{\gamma}}\left(-c_{2}+c_{1} g\right)$, i.e. when the steady state is reached $c_{2}$ is either 1 or a value that satisfies $g=c_{2} / c_{1}$ where $g\left(c_{2}\right):=\frac{1+\beta}{1+\beta-c_{2}}$. In the top subplot of Fig. 5, the parabolic (lower) curve depicts the solution obtained from $-c_{2}+c_{1} g=0$. The same subplot also depicts the value of steady state control action $w_{s s}$. This figure stipulates that when the steady state is reached, the cell mass cannot assume values larger than $\frac{1+\beta}{4}=0.255$. This practical constraint was also highlighted in [3] and [4]. The bottom subplot of Fig. 5 depicts how an arbitrary value of $\left(c_{1}\left(t_{0}\right), c_{2}\left(t_{0}\right)\right)$ moves under the control action $w_{s s}$. Few comments can be made for the motion for $t>t_{0}$ with $w_{s s}$. If $c_{1}\left(t_{0}\right)>\frac{1+\beta}{4}$ then the final value of the state vector is $\left(c_{1}(\infty), c_{2}(\infty)\right)=$ 

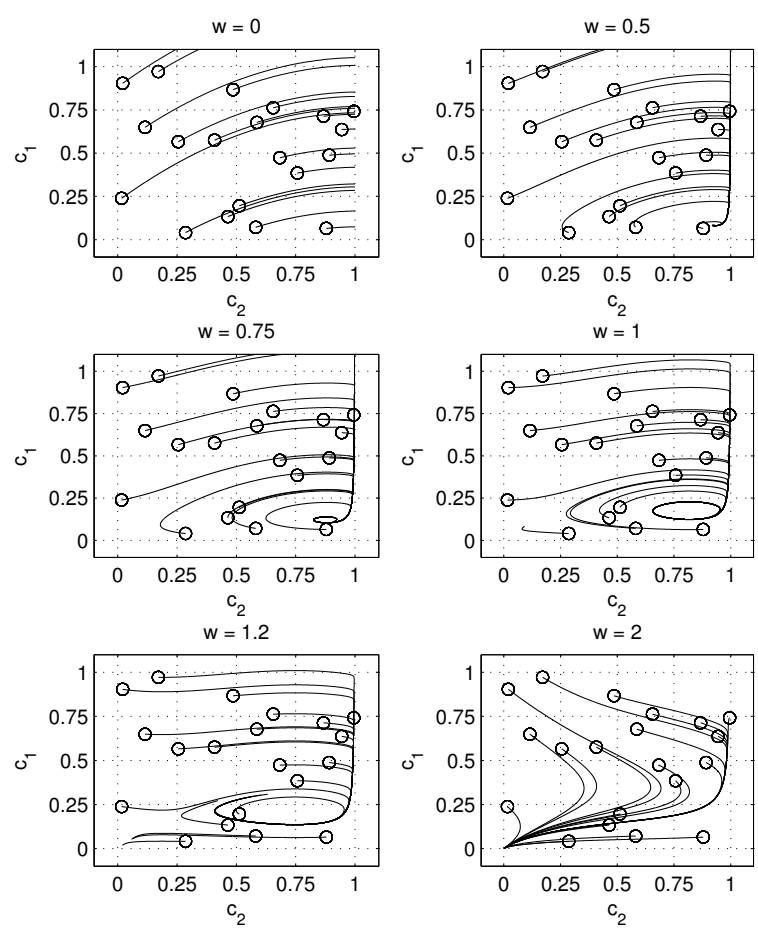

Fig. 2. The evolution of the process state for different initial conditions and at different inflow rates. The trajectories are for 20 seconds time.

$\left(c_{1}\left(t_{0}\right), 1\right)$. If $c_{1}\left(t_{0}\right)<\frac{1+\beta}{4}$, then any initial condition that is below the curve defined by $-c_{2}+c_{1} g=0$ moves in the horizontal direction and stops on the left segment of the curve $-c_{2}+c_{1} g=0$, which is shown in the bottom subplot of Fig. 5. Alternatively, the initial conditions that are above the curve defined by $-c_{2}+c_{1} g=0$ move right until an equilibrium is reached, i.e. those satisfying $c_{2}\left(t_{0}\right)<\frac{1+\beta}{2}$ converge the left segment of the curve $-c_{2}+c_{1} g=0$, however those with $c_{2}\left(t_{0}\right)>\frac{1+\beta}{2}$ stop at $\left(c_{1}(\infty), c_{2}(\infty)\right)=\left(c_{1}\left(t_{0}\right), 1\right)$. At the point $\left(c_{1}\left(t_{0}\right), c_{2}\left(t_{0}\right)\right)=\left(\frac{1+\beta}{4}, \frac{1+\beta}{2}\right)$, the eigenvalues of the linearized system are both equal to zero. According to the behavior indicated by the vector field (flow), we conclude that this point is a half stable point, i.e. the trajectories close to this point but below $-c_{2}+c_{1} g=0$ behave different from those above $-c_{2}+c_{1} g=0$. This discussion with the flow illustrated in Fig. 5 clarifies the stability of the equilibrium states thoroughly.

A last point that should be emphasized is the effect of sampling in the control loop. In [2], the equation system in (1) is discretized by Euler method and a step size $\Delta=0.01$ sec. is used. The sampling period for the control signal, called macro time steps ([3]) is equal to $50 \Delta$ sec., which is long enough for the bioreactor process to develop deviations and spontaneous oscillations from a desired setpoint or a trajectory. In this paper, we test the performance of the proposed controller by operating it at the same rates too.

In [2], Ungar points out that although this system is

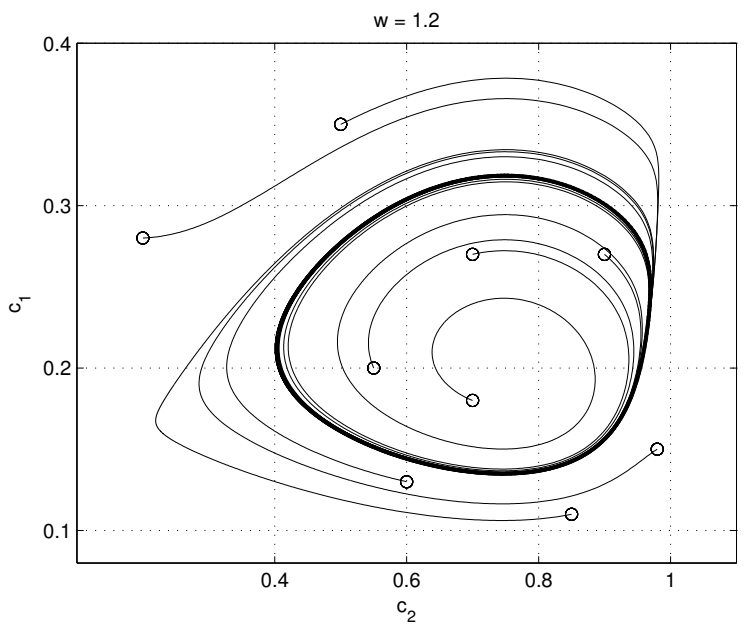

Fig. 3. Limit cycle arising when $w=1.2$

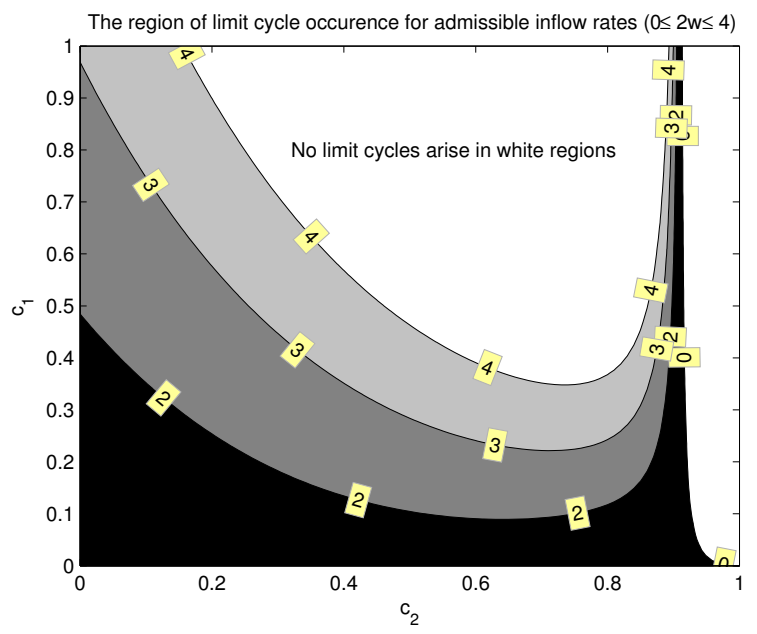

Fig. 4. Regions where limit cycles cannot occur are designated by white color

not a completely realistic model of any bioreactor, as seen from the presented discussion, the system considered in this paper displays several challenges highlighted also by [7] with a similar motivation. Due to the presented properties of the system, the model constitutes a good candidate for scrutinizing the merits and effectiveness of nonlinear control laws.

\section{Sliding Mode CONTROL OF THE PROCESS}

$$
\begin{gathered}
\text { Let } f:=c_{1}\left(1-c_{2}\right) e^{\frac{c_{2}}{\gamma}} \text { and } g:=\frac{1+\beta}{1+\beta-c_{2}} \text {. Let } \\
\dot{c}_{1 m}=-\lambda\left(c_{1 m}-r\right), \quad \lambda>0
\end{gathered}
$$

be the reference model and denote the command signal by $r$. Defining $s:=c_{1}-c_{1 m}$ as the switching variable, the control law given by

$$
w=\frac{1}{c_{1}}\left(f+\lambda\left(c_{1 m}-r\right)+\zeta \operatorname{sgn}(s)+\eta s\right)
$$



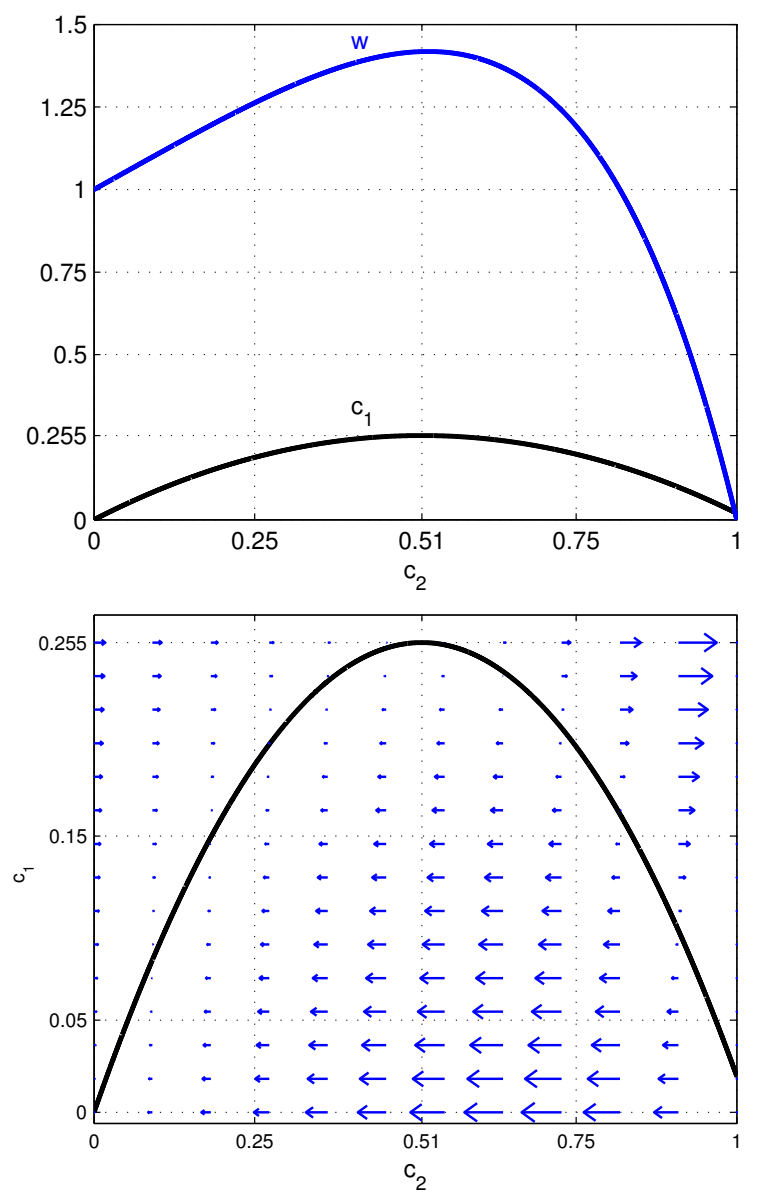

Fig. 5. Equilibrium points and the behavior with steady state control signal $w_{s s}$

ensures $\dot{s}=-\zeta \operatorname{sgn}(s)-\eta s$, which renders the system against the attractors and repellers, if any. The control law above it sure that the cell mass reaches the model output in finite time satisfying $t_{h}<\frac{|s(0)|}{\zeta}$ and results in

$$
\dot{c}_{2}=\left(g-\frac{c_{2}}{c_{1}}\right) f-\frac{c_{2}}{c_{1}}\left(\lambda\left(c_{1 m}-r\right)+\zeta \operatorname{sgn}(s)+\eta s\right)
$$

in the closed loop. Clearly, the desired cell mass is reached when $t=t_{h}$, indicating $s=0$. For $t \geq t_{h}, s=0$ is maintained, i.e. $c_{1}=c_{1 m}$ and the following equalities can be written;

$$
\dot{c}_{2}=\left(g-\frac{c_{2}}{c_{1 m}}\right) f+\frac{c_{2}}{c_{1 m}} \dot{c}_{1 m}
$$

or alternatively,

$$
\begin{aligned}
& \frac{\dot{c}_{2} c_{1 m}-\dot{c}_{1 m} c_{2}}{c_{1 m}^{2}}=\frac{1}{c_{1 m}}\left(g-\frac{c_{2}}{c_{1 m}}\right) f \\
& \frac{\mathrm{d}}{\mathrm{d} t}\left(\frac{c_{2}}{c_{1 m}}\right)=\left(g-\frac{c_{2}}{c_{1 m}}\right)\left(1-c_{2}\right) e^{\frac{c_{2}}{\gamma}}
\end{aligned}
$$

Defining $u:=\frac{c_{2}}{c_{1 m}}$ as the ratio of nutrients per unit of (prescribed) cell mass, the equality in (9) can be rewritten compactly as

$$
\dot{u}=(g-u)\left(1-c_{2}\right) e^{\frac{c_{2}}{\gamma}}
$$

According to the Fig. 5, the equilibrium value of (10) is a global attractor within $\Omega$ and a useful set of equilibria is reached only when $u=g$ holds true.

Recall that the expression $\left(1-c_{2}\right) e^{\frac{c_{2}}{\gamma}}$ was termed $w_{s s}$, the steady state control action. Rewriting (10) as

$$
\dot{u}=-w_{s s} u+w_{s s} g
$$

shows that $u$ is forced to follow $g$ as long as $0 \leq c_{2}<1$ holds true. This conclusion is due to the nonnegativeness of $w_{s s}$. If $c_{2}=1$ for some time, then $w_{s s}=0$ and $\dot{u}=0$, i.e. no change in $u$ occurs as long as $c_{2}=1$ is satisfied. But any nonzero inflow rate causes $0 \leq c_{2}<1$, the condition of the theorem is satisfied, and $u$ settles down to a steady state value determined by $u=g$.

In Fig. 6, several values of $\dot{u}$ are contoured over a vector field. For every value of $c_{2}$, the illustrated vector field illustrated indicates that $u$ tends to converge to a constant value on the thick contour labeled 0 in the figure. This curve describes the steady state $\dot{u}=(g-u)\left(1-c_{2}\right) e^{\frac{c_{2}}{\gamma}}=0$, and is a global attractor within $\Omega$. We visualize the behavior for $0 \leq u \leq 15$ for better illustration. Due to the smoothness of the involved expressions, the same behavior is seen for large values of $u$ too.

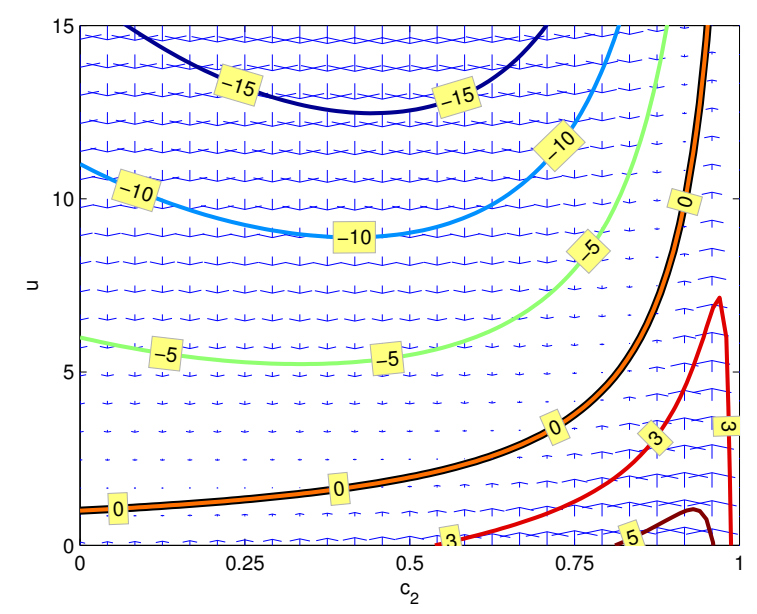

Fig. 6. Qualitative behavior of $u$ versus $c_{2}$

\section{Simulation Studies}

The control law in (5) is realized under the following operating conditions.

- The observations for the state variables $c_{1}(t)$ and $c_{2}(t)$ are noisy. The Gaussian noise sequences corrupting the state variables lie within the interval $[-0.003,0.003]$ with a probability very close to unity. The noise in the observations is a difficulty introducing uncertainties that 
necessitates the design and implementation of a robust controller.

- In [2], it is emphasized that small variations in the values of $\gamma$ and $\beta$ lead to significant deviations from the target cell mass. For example, given perfect measurements, $2 \%$ change in $\gamma$ and $20 \%$ change in $\beta$ may cause $50 \%$ deviation in the cell mass, $c_{1}(t)$, (See [2], [6]). In this paper, we consider the nominal values of these parameters for the design of the controller and in the justification of the proposed scheme, we study these parameters with some variation in time. The necessity for investigating the behavior under parameter variations is tightly relevant to the need of exploring the controller performance under extreme conditions. The value of $\gamma$ changes within the interval $[0.4634,0.4966]$, which means maximum $3.47 \%$ change in the nutrient inhibition parameter. Likewise, $\beta$ displays a variation in $[0.1276,3.5717] \times 10^{-2}$ indicating a maximum of $93.62 \%$ deviation from the nominal value given by $\beta=0.02$. Clearly the chosen profiles for the variables and the presence of measurement noise require certain degrees of robustness to meet the stability and performance requirements.

- Another difficulty is the large initial error in the cell mass. If the initial value of $e_{1}$ is large, then the controller must force it towards zero with a sequence of admissible inflow rates, $w(t)$, and must maintain the stability during the transient phase. This paper also addresses the issue of handling the large initial errors. In the simulations, we have chosen $c_{1 m}(0)=0, c_{1}(0)=$ $0.4, c_{2}(0)=0.7$ and $r(0)=0.0175$, which perfectly enable us to demonstrate the effect of the initial errors.

- The choice of the reference signal is another important issue in closed loop control. The desired cell mass (dashed curve) claims the tracking of a discontinuous desired profile to see how the controller stabilizes the system. The command profile studied in this paper enables us to figure out the qualitative and quantitative observations arise during the reaching phase especially at different cell mass $\left(c_{1}(t)\right)$ levels.

- In order to avoid the undesired chattering phenomenon arising because of the measurement of a quantity, which is very close to zero, we use $\operatorname{sgn}(s) \approx \frac{s}{|s|+\delta}$, with $\delta=$ 0.05 .

- Finally, the effect of actuation interval for the controller will be emphasized. Regarding this issue, [2] defines $T=50 \Delta=0.5 \mathrm{sec}$. as the control interval. In other words, the inflow rate maintains its value during $n T \leq t<(n+1) T$, where $n$ is a discrete time index. Consequently, the computation of the control signal applied during this interval is based on the observations at $t=n T$. The practical drawback of such an actuation scheme is the following: As discussed in the second section and shown in Fig. 2, the system may get trapped to a limit cycle or an attractor during this time and this makes it necessary to implement a perfect flow rate management strategy.
The other parameters of the controller are $\zeta=0.05$ and $\eta=0.1$, which have been set after few trials. The reference model has $\lambda=0.1$. As shown in the top subplot of Fig. 7, the cell mass (solid curve) closely follows the desired cell mass profile (dashed curve). The middle subplot depicts the discrepancy between the process response and the model output, i.e. $e_{1}(t)=c_{1}(t)-c_{1 m}(t)$, or in other words, the value of the switching function, $s(t)$. The results seen emphasize that very small model following error is maintained. The bottom subplot of the figure depicts the evolution of nutrient amount, $c_{2}(t)$. The variable evolves bounded as claimed in the derivation of the controller. The initial transient regime in the cell mass is illustrated in a window at the bottom of the figure, where it is convincingly apparent that hitting occurs around $t_{h}=9.4 \mathrm{sec}$. The theoretical value is $t_{h} \leq \frac{|s(0)|}{\zeta} \approx 8 \mathrm{sec}$. since $s(0)=0.4$.

A substantially important measure for practical applicability is the cost of physical realizability of the signals produced by the controller. The time evolution of the inflow rate corresponding to the emergence of the behavior shown in Fig. 7 is illustrated in Fig. 8. Clearly the signal is smooth enough to realize as shown also in the window plots. It is worthwhile to stress that the control signal is saturated for a very short while only when there are step changes in the command signal. During the rest of the operation, the control signal is smooth enough and the fluctuations are convergent. These prominent features of the control signal demonstrate that the controller possesses highly desirable characteristics such as good disturbance rejection capability and guaranteed tracking precision under the presence of uncertainties. The use of sign function smoothing introduces a thin boundary layer whose thickness is determined by $\delta$, [16]. If $s(t)$ is within the boundary layer, then it approaches the origin smoothly. This modification of the original control law gives a very slight rise to the value of $t_{h}$, which is found to be acceptable. From a practical point of view, smoothness of the control signal makes it possible to implement the control law with hardware having no or little extraordinary properties at the cost of giving very slight concessions from the performance.

A last quantity to monitor is given by $g-\frac{c_{2}}{c_{1}}$, which is forced to zero. Referring to Fig. 9, we have shown that the control law in (5) forces all trajectories shown in Fig. 6 to the locus described by $g-\frac{c_{2}}{c_{1}}=0$, which explains the result shown in Fig. 9. The window plot in the figure depicts the initial transient regime, which very quickly converges to zero and the motion thereafter takes place in the vicinity of zero, i.e. $g-\frac{c_{2}}{c_{1}} \approx 0$ is maintained.

\section{CONCLUSIONS}

Model reference sliding mode control of a biochemical process, introduced as a benchmark problem, is considered. Limitations and the dynamical properties of the process are studied, then a first order reference model is chosen and the cell mass, which is one of the two state variables, is asked to follow the output of the chosen reference model. The discontinuous control law enforcing the sliding regime 

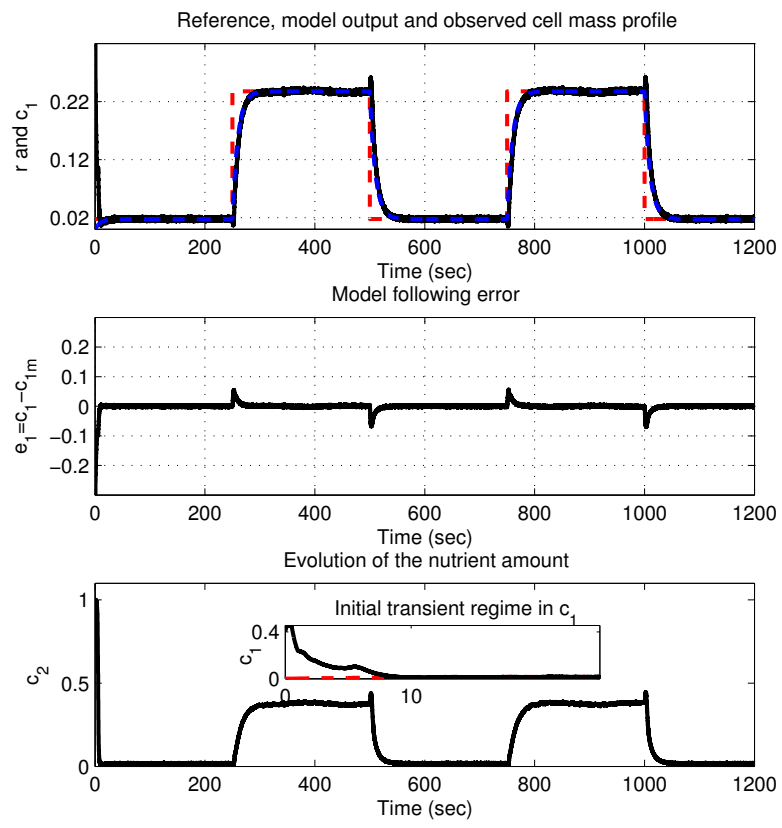

Fig. 7. The evolution of the state variables

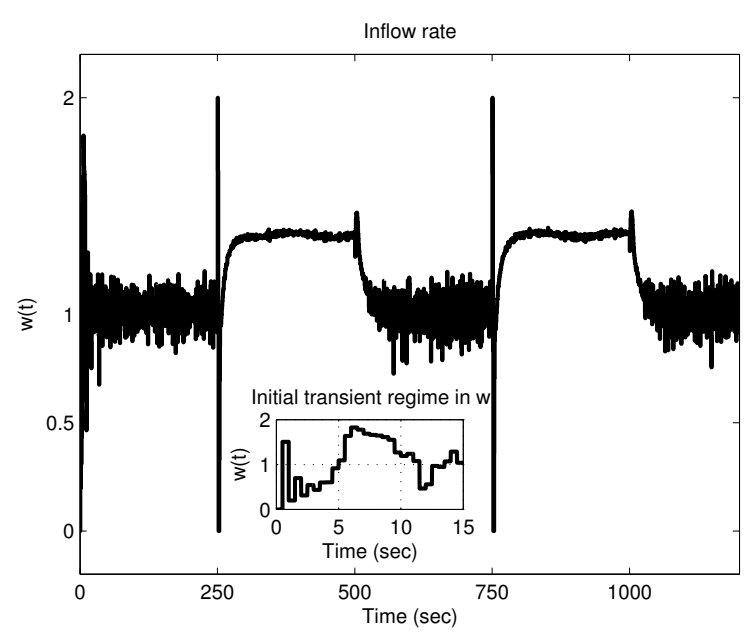

Fig. 8. The evolution of the inflow rate

has been shown to result in bounded evolution in the nutrient amount, which is the other state variable. The closed loop control system has been tested under the presence of several difficulties. Namely, the large initial errors, time varying process parameters, observation noise are those deserving emphasis. The controller is observed to be capable of driving the cell mass to its desired value prescribed by the reference model quickly and maintaining the model following accuracy.

\section{ACKNOWLEDGMents}

This work was supported in part Turkish Scientific Council (TÜBITAK) Contract 107E137 and by TOBB Economics

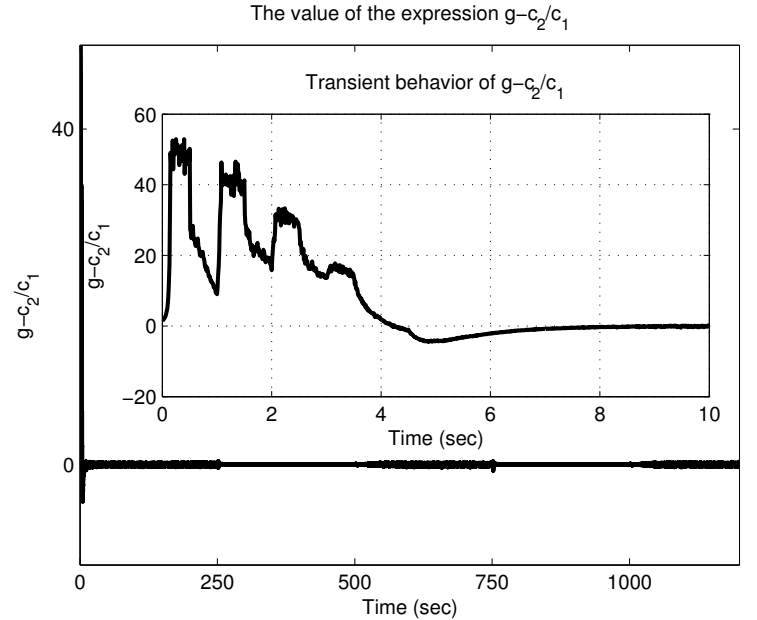

Fig. 9. The evolution of the variable $g-\frac{c_{2}}{c_{1}}$

and Technology University, BAP Program, under contract no ETÜ-BAP-2006/04.

\section{REFERENCES}

[1] Bequette, B.W. (1991), "Nonlinear Control of Chemical Processes: A Review,” Ind. Eng. Chem. Res., v.30 pp.1391-1413.

[2] Ungar, L. H. (1990), "A Bioreactor Benchmark for Adaptive-Network Based Process Control," Neural Networks for Control, W. T. Miller III, R. S. Sutton, P. J. Werbos, Eds, MIT Press, pp.387-402.

[3] Puskorius, G.V., Feldkamp, L.A. (1990), "Neurocontrol of Nonlinear Dynamical Systems with Kalman Filter Trained Recurrent Networks," IEEE Trans. on Neural Networks, v.5, n.2, pp.279-297.

[4] Efe, M.Ö., Abadoglu, E. and Kaynak, O. (1999), "A Novel Analysis and Design of a Neural Network Assisted Nonlinear Controller for a Bioreactor," Int. Journal of Robust and Nonlinear Control, v.9, no.11, pp.799-815.

[5] Efe, M.Ö. (2007) "MIMO Variable Structure Controller Design for a Bioreactor Benchmark Process," ISA Transactions, v.46, pp.459-469.

[6] Brengel, D.D. and Seider, W.D. (1989), "Multistep Nonlinear Predictive Controller," Ind. Eng. Chem. Res., v.28, pp.1812-1822.

[7] Anderson, C. W. and W. T. Miller III (1990), "Challenging Control Problems", Neural Networks for Control, W. T. Miller III, R. S. Sutton, P. J. Werbos, Eds, MIT Press, pp.475-510.

[8] Hung, J. Y., W. Gao and J. C. Hung (1993), "Variable Structure Control: A Survey," IEEE Transactions on Industrial Electronics, v.40, n.1, pp.222.

[9] Utkin, V.I., Sliding Modes in Control Optimization, Springer Verlag, New York, (1992).

[10] Hanczyc, E.M. and Palazoglu, A. (1995), "Sliding Mode Control of Nonlinear Distributed Parameter Chemical Processes," Ind. Eng. Chem. Res., v.34, pp.557-566.

[11] Camacho, O. and Rojas, R. (2000), "A General Sliding Mode Controller for Nonlinear Chemical Processes," Trans. of the ASME: Journal of Dynamic Systems, Measurement and Control, v.122, pp.650-655.

[12] Colantonio, M.C., Desages, A.C., Romagnoli, J.A. and Palazoglu, A. (1995), "Nonlinear Control of a CSTR: Disturbance Rejection Using Sliding Mode Control,” Ind. Eng. Chem. Res., v.34, pp.2383-2392.

[13] O. Kaynak, Harashima, F. and Hashimoto, H. (1984), "Variable Structure Systems Theory, as Applied to Sub-time Optimal Position Control With an Invariant Trajectory," Trans. Inst. Elect. Eng. Jpn. E, 104, pp. 4752 .

[14] Slotine, J.-J.E. and Li, W. (1991), Applied Nonlinear Control, Prentice Hall, New Jersey.

[15] Khalil, H. (2001), Nonlinear Systems, Prentice Hall, New Jersey.

[16] Young, K. D., V. I. Utkin, and Ü. Özgüner (1999), "A Control Engineer's Guide to Sliding Mode Control," IEEE Transactions on Control Systems Technology, v.7, n.3, pp. 328-342. 